\title{
Captiver/séduire. Note sur la gestuelle des joueurs de hautbois nāgasvaram
}

Seduce/charm. Note on the gesture of nāgasvaram shawm players

\section{William Tallotte}

\section{(2) OpenEdition}

Journals

Édition électronique

URL : http://journals.openedition.org/transposition/339

DOI : 10.4000/transposition.339

ISSN : 2110-6134

Éditeur

CRAL - Centre de recherche sur les arts et le langage

Référence électronique

William Tallotte, "Captiver/séduire. Note sur la gestuelle des joueurs de hautbois nāgasvaram », Transposition [En ligne], 2 | 2012, mis en ligne le 01 mai 2012, consulté le 30 juillet 2019. URL : http:// journals.openedition.org/transposition/339; DOI : 10.4000/transposition.339

Ce document a été généré automatiquement le 30 juillet 2019.

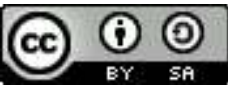

La revue Transposition est mise à disposition selon les termes de la Licence Creative Commons Attribution - Partage dans les Mêmes Conditions 4.0 International. 


\section{Captiver/séduire. Note sur la gestuelle des joueurs de hautbois nāgasvaram}

Seduce/charm. Note on the gesture of nägasvaram shawm players

William Tallotte

1 Outre des techniques de souffle, de pince, de langue et de doigts à la fois exigeantes et complexes, le jeu du hautbois nāgasvaram ${ }^{1}$ fait appel à des gestes qui, emmenés par les bras, les épaules et le buste, offrent aux musiciens la possibilité de mettre en mouvement leur instrument: verticalement, latéralement, ou en combinant ces deux plans. Ces gestes, ces mouvements, n'interviennent pas directement dans la production du son. Ils ne marquent pas non plus physiquement l'instrument - contrairement aux gestes digitaux qui, avec le temps, lissent, creusent et décolorent le contour des trous de jeu; contrairement aux gestes buccaux qui, via le contact répété des lèvres, des dents, de la salive, rongent peu à peu le roseau des anches. Non, rien de cela dans ces gestes auxiliaires ${ }^{2}$, extra- ou para-musicaux : seulement des figures éphémères qui frappent l'imagination le temps d'une performance et que l'on garde vaguement - ou non - en mémoire.

2 Je tenterai dans ce texte - dans la continuité de travaux récents menés sur le geste musical $^{3}$ - de répondre aux questions suivantes: ces gestes ou mouvements sont-ils codifiés ? Ont-ils pour seule fonction, comme le laissent parfois entendre les musiciens, de captiver/séduire un auditoire? Enfin, sont-ils sensibles aux contextes et/ou aux configurations acoustiques?

\section{Positions types et mouvements latéraux}

3 L'observation in situ, l'apprentissage de l'instrument, la fréquentation des musiciens, le dépouillement des archives, permettent d'identifier trois positions types (1,2 et 3), lesquelles peuvent être adaptées au jeu debout (a) ou assis en tailleur (b) ${ }^{4}$ : 


\begin{tabular}{|l|l|l|}
\hline & Debout (a) quasiment & Assis en tailleur (b) \\
\hline Position basse (1) & $\begin{array}{l}\text { Hautbois } \\
\text { parallèle au corps qui repose sur le sol (fig. 3) }\end{array}$ \\
\hline $\begin{array}{l}\text { Position } \\
\text { intermédiaire (2) }\end{array}$ & Inclinaison moyenne & $\begin{array}{l}\text { Inclinaison moyenne, sans que le pavillon } \\
\text { touche le sol (fig. 1, 2 et 3) }\end{array}$ \\
\hline Position haute (3) & $\begin{array}{l}\text { Hautbois perpendiculaire } \\
\text { au corps }\end{array}$ & Hautbois perpendiculaire au corps (fig. 4) \\
\hline
\end{tabular}

4 Ces positions, $1 \mathrm{a}, 1 \mathrm{~b}, 2 \mathrm{a}, 2 \mathrm{~b}, 3 \mathrm{a}$ et $3 \mathrm{~b}$, offrent une première indication : le hautbois ne peut être levé au-dessus d'un certain niveau, c'est-à-dire perpendiculairement au corps (fig. 4). Pourquoi ? Une première réponse renvoie au contrôle du souffle. Un jeu fluide, doublé d'une belle sonorité, nécessite une assise solide et un bon maintien de la « colonne d'air ». Ce bon maintien est encouragé lors de l'apprentissage à travers quelques conseils: "respire/souffle par le ventre", "relâche tes épaules", "reste droit», "garde ta colonne (vertébrale) bien droite... », " ...afin que l'air passe tout droit de ton nombril à ta bouche ", ce que l'on pourrait encore traduire par "garde ta colonne (d'air) bien droite » - formule certes curieuse d'un point de vue physiologique mais néanmoins approchante... et suggestive : elle évoque, en effet, la figure de l'ascète hindou et, plus concrètement, un ensemble de pratiques de rétention du souffle où, justement, une position droite du dos et de la colonne est recherchée, travaillée et sans cesse affinée dans le but d'ouvrir progressivement les soupapes d'une "colonne d'air " interne qui monterait en ligne droite du bas ventre jusqu'à la tête. On comprend dès lors - c'est au moins l'argument des musiciens - que si le hautbois est levé au-dessus de la position 3, la nuque est alors portée en arrière et vient former un angle qui peut nuire à la qualité et la puissance d'émission $\mathrm{du}$ souffle. On relèvera cependant une exception: celle du hautboïste Injikkudi M. Subramaniam qui, en position assise (b), utilise un petit basculement arrière lui permettant de signifier un au-delà de la position haute type ( $3 b)$ en conservant un angle 
droit parfait entre son corps et son instrument tout en élevant légèrement la direction du pavillon (fig. 5).

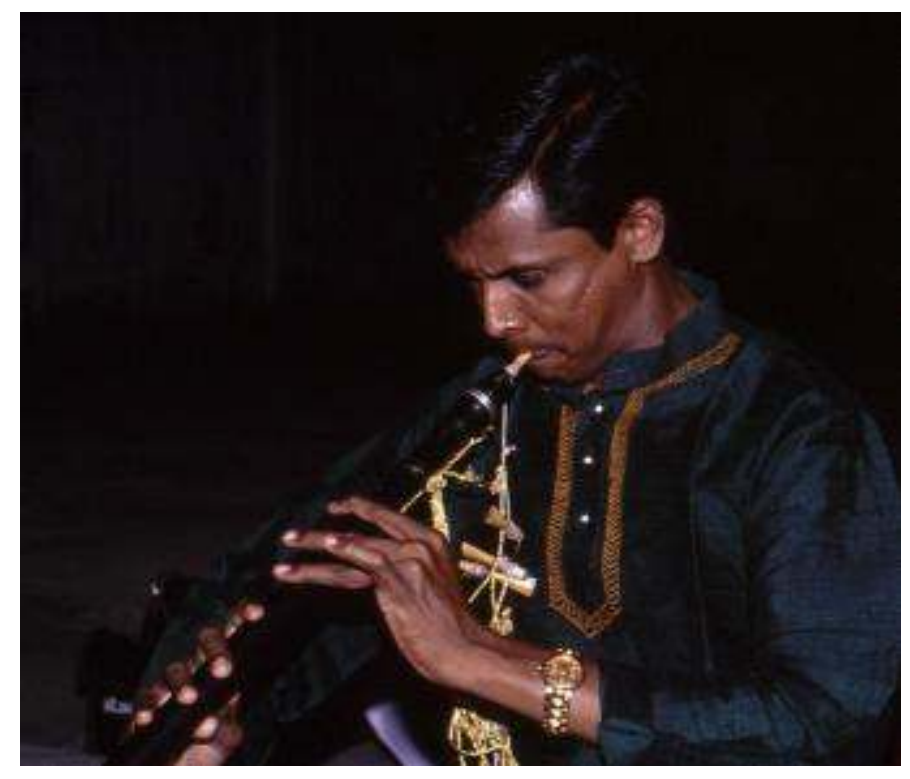

fig. 1

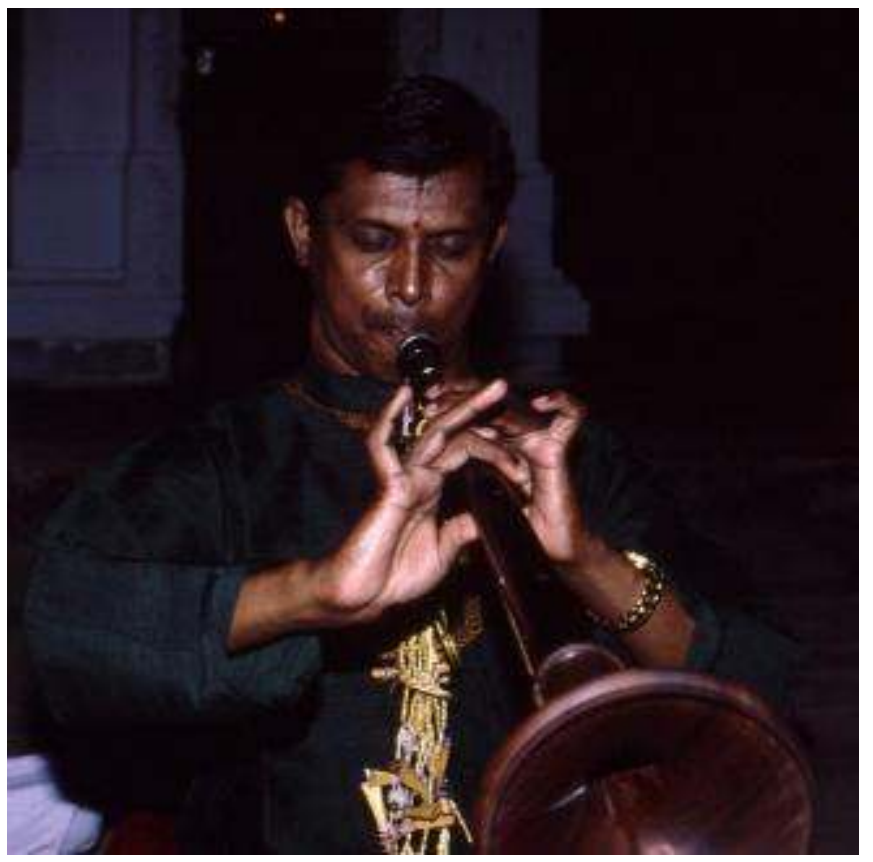

fig. 2 


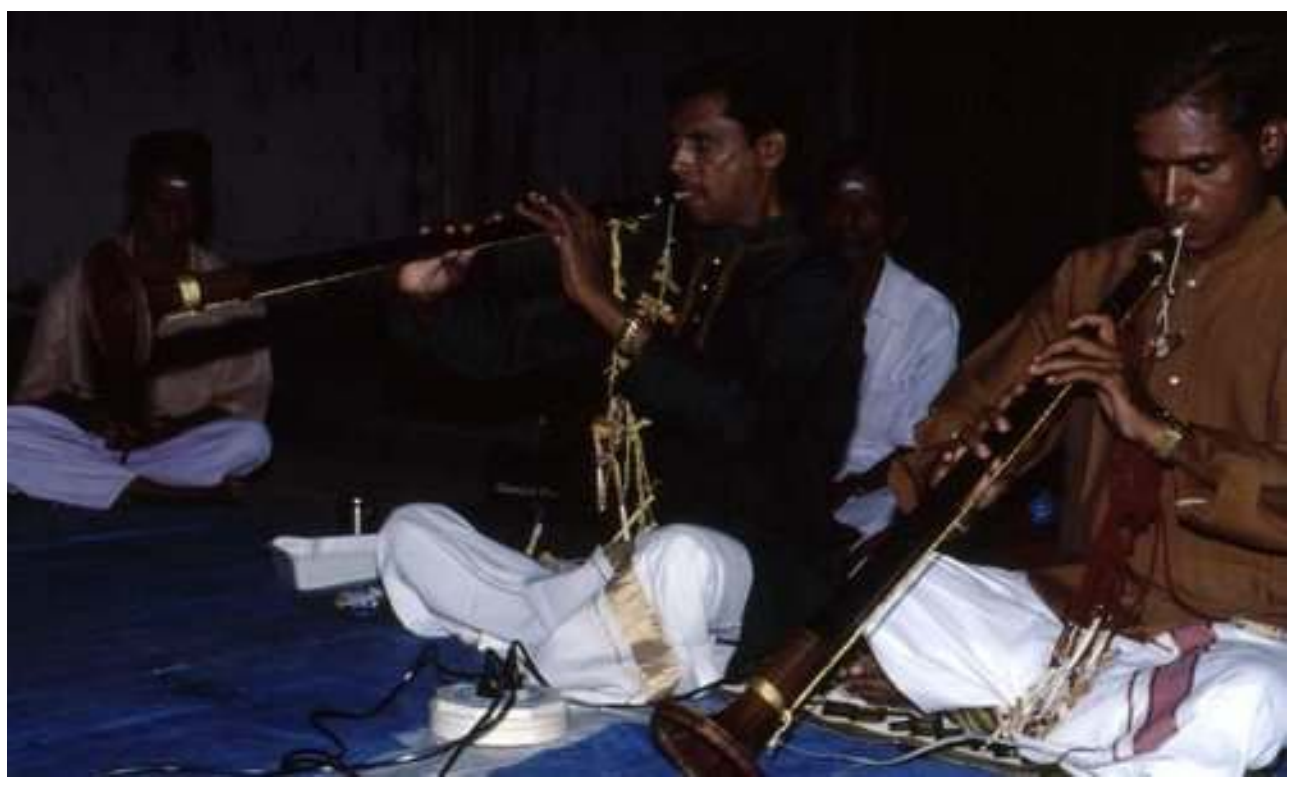

fig. 3

fig. 1, 2 et 3. Injikkudi M. Subramaniam en positon 2b et selon des inclinaisons variables, plus ou moins proches des positions 1b (fig. 1) ou 3b (fig. 2 et 3) ; et Kumbakonam V.R. Muruganandan en position 1b (fig. 3). Photos William Tallotte, Tirukkadaiyur, 2005.

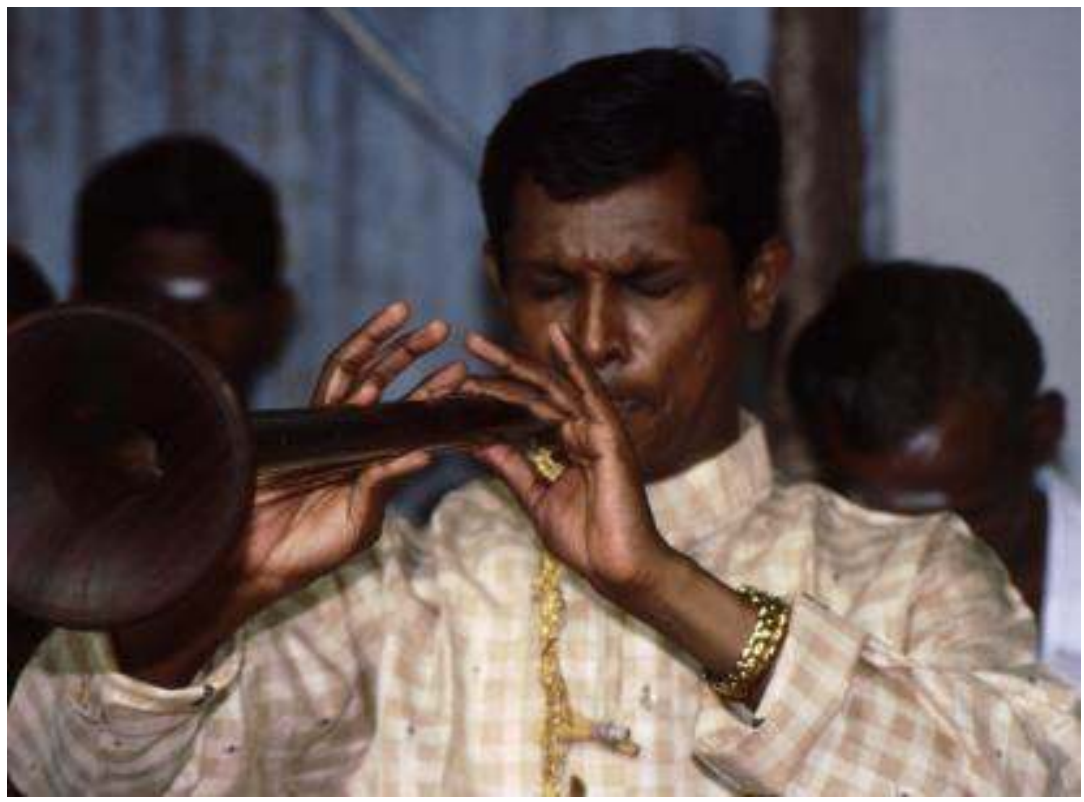

fig. 4 


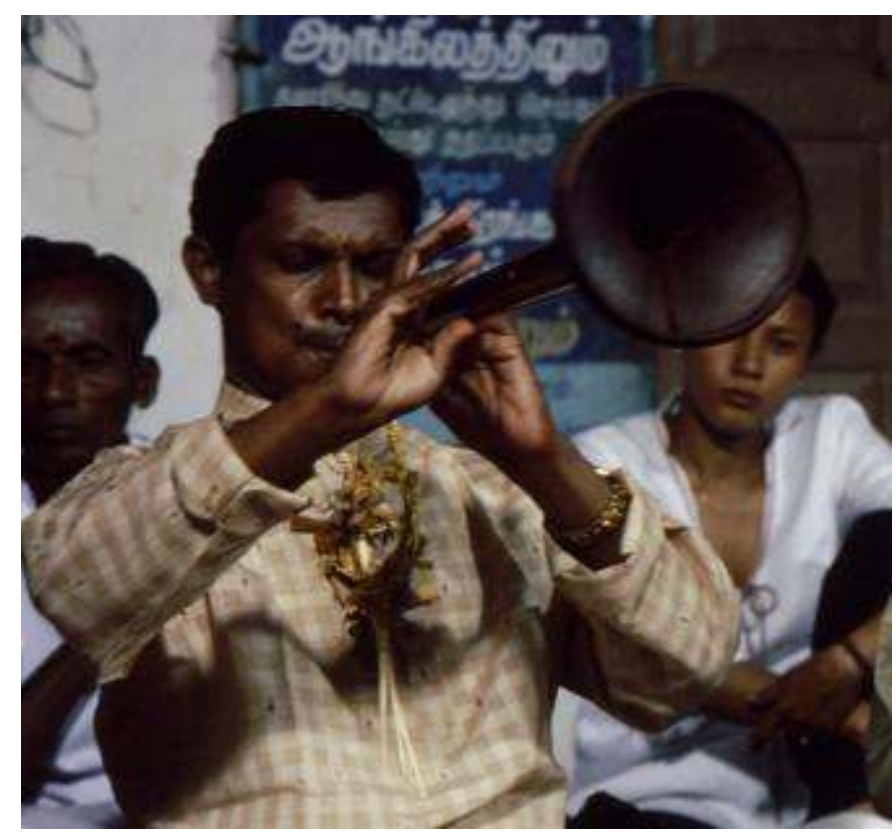

fig. 5

fig. 4 et 5. Injikkudi M. Subramaniam en position 3b (fig. 4), puis au-delà de la position 3b (fig. 5). Photos William Tallotte, Pondichéry, 2005.

5 Une deuxième réponse renvoie aux contextes de jeu et au besoin qu'éprouvent les joueurs de nāgasvaram de se démarquer des hautboïstes d'une tradition de sonneurs-batteurs - le naiyāndi mẹlam ${ }^{5}$ (" orchestre de raillerie ») - qui utilisent le même hautbois mais dont les attitudes corporelles, liées à la transe et aux cultes de possession des temples de basses castes, sont bien plus extraverties et loin, en conséquence, de l'image sereine et imperturbable du yogi en position de méditation ${ }^{6}$.

6 Au-delà de ces positions et des mouvements verticaux qu'elles impliquent - de 1 à 2 ou à 3 , de 2 à 1 ou à 3 , de 3 à 2 ou à 1 - se pose bien sûr la question des mouvements latéraux. L'analyse des données, notes de terrain, vidéos, photographies, montre que l'amplitude de ces mouvements varie considérablement d'un musicien à l'autre: en fonction de sa lignée, des contextes de jeu qu'il fréquente, voire de son propre caractère, plus ou moins expansif, exubérant, démonstratif. Mais qu'on ne s'y trompe pas: cette liberté est encadrée par des règles plus ou moins tacites :

7 L'amplitude latérale maximum est de 70-80 degrés environ, à gauche comme à droite ; elle ne peut être observée qu'en position 1a, $3 a$ et $3 b$. La plupart des musiciens s'en tiennent cependant à un balayage plus serré de l'espace, de 30 à 40 degrés environ.

8 Assis en tailleur (b), l'amplitude augmente latéralement des positions 1 à 3 et donc progressivement du bas vers le haut.

Debout (a), ce phénomène d'expansion ne fonctionne que des positions 2 à 3 puisque des mouvements latéraux peuvent être effectués en position 1a - ce qui n'est pas le cas en position $1 \mathrm{~b}$ où le pavillon repose sur le sol. Notons toutefois que si la rotation du buste en position 1a est comparable à celle effectuée en position 3a, voire $3 b$, l'effet visuel est moins saisissant puisque l'espace parcouru par le pavillon est à son minimum lorsque le hautbois est parallèle au corps, à son maximum lorsqu'il est perpendiculaire au corps. 


$$
\text { en particulier dans des contextes - telles les fêtes de temple - où celles-ci sont }
$$
longuement développées.

11 Les gestes auxiliaires des joueurs de nāgasvaram répondent donc à des limites spatiales qu'il est préférable de respecter - sous peine d'être sévèrement critiqué par ses pairs. À l'intérieur de ces limites, l'expression individuelle semble néanmoins respectée, sinon encouragée. Du moins n'est-elle jamais réprouvée : son originalité va seulement de soi, à l'instar d'une façon de marcher, à la fois identique à toutes les autres et reconnaissable entre toutes. À chacun sa manière de combiner tels mouvements, de passer de l'un à l'autre, de tracer dans l'espace ses propres figures : lignes qui se croisent, ondulent ou se superposent, courbes ouvertes ou fermées, ponctuées ou non de traits incisifs. On peut cependant se poser la question suivante : pourquoi, à cet instant, là précisément, ce geste plutôt qu'un autre?

\section{Captiver, séduire... le point de vue des musiciens}

Bien que cette gestuelle soit observable partout, au temple comme au concert, personne ne s'y intéresse. Elle ne fait l'objet - à ma connaissance au moins - d'aucun commentaire, d'aucune verbalisation : ni dans les textes (traités, études, articles de presse, etc.) ni dans les discussions (entre musiciens, connaisseurs, musiciens et connaisseurs, etc.). Pour autant, l'expérience du terrain, en l'occurrence la fréquentation régulière des musiciens, laisse des indices sur la ou les raison(s) de ces gestes. Deux d'entre eux me semblent plus particulièrement explicites :

\section{Premier indice}

13 Chidambaram, début 2001. Je me rends trois à quatre fois par semaine chez le joueur de nāgasvaram S.R.D. Vaidyanathan ${ }^{7}$. Le déroulement des cours suit grosso modo ce modèle : je répète en boucle les mêmes exercices tandis que Vaidyanathan, tout en gardant une oreille alerte, vaque à ses occupations. À la moindre erreur, il déboule d'une pièce voisine et me chante la séquence que j'ai escamotée et la mime de ses mains - ses doigts sont dans le vide, au dessus d'un instrument (d'un corps) imaginaire. De temps en temps, à ma demande, cette routine est brisée. Vaidyanathan prend alors son hautbois et, face à moi, m'initie à la forme âlāpana - forme improvisée, non mesurée, au rythme libre, dans laquelle un mode (rāga) est développé. C'est dans ce cadre, précisément, qu'il m'encourage parfois à lever mon hautbois afin d'exécuter d'amples mouvements latéraux. S'il est satisfait, il dresse son pouce à hauteur de visage en secouant légèrement sa main ou, autre possibilité, pointe son index vers le ciel en traçant des cercles dans le sens inverse des aiguilles d'une montre. J'ai toujours interprété ces signes de la manière suivante : mouvoir ainsi son instrument permettrait non seulement de capter l'attention des auditeurs et donc de les impressionner (signe du pouce), mais de s'imposer comme un musicien de premier plan - un musicien qui « tourne » (signe de l'index).

\section{Deuxième indice}

Mayuram, mars 2008. Je discute avec le joueur de hautbois Injikkudi P. Ganeshan. Je lance un sujet : l'improvisation musicale. Ganeshan en vient à parler des gestes qu'il exécute 
afin de mouvoir son instrument verticalement : selon lui, lever son hautbois relèverait d'un acte de communication, voire de séduction. L'analogie qu'il donne est éloquente :

Quand je joue un ālāpana, je suis attentif aux réactions du public. Lorsque je m'engage dans un registre plus aigu, je commence à observer, à écouter. Je repose de moins en moins le pavillon sur le sol. Le moment venu, quand je sens que le public est avec moi, je lève le hautbois. C'est un peu comme quand tu entres sur le territoire d'un cobra (nāga), alors il se dresse. Il veut t'impressionner. Moi c'est pareil, mais je ne cherche qu'à séduire ${ }^{8}$.

Ces deux indices se recoupent et laissent supposer que ces gestes auxiliaires ont non seulement pour but de captiver, mais de séduire les auditeurs - également spectateurs. Tentons d'aller plus loin.

\section{Captiver, séduire... mais encore : essai de typologie}

Captiver/séduire : est-ce bien la seule, sinon la principale fonction de ces mouvements? Par ailleurs, ces mouvements sont-ils connectés à la musique, à son contenu, à son expressivité ? Permettent-ils de souligner ou de mettre en avant une séquence, une phrase, un motif ? Bref, relèvent-ils d'un acte - conscient ou non - de communication ? Ces questions sont épineuses, et pour cause : elles renvoient à des savoir-faire et des conduites non seulement incorporées mais implicites. Il serait donc vain, me semble-t-il, de recourir à des entretiens, et qui plus est des entretiens directifs, alors même que les intéressés ne s'expriment pas naturellement sur ce point. À moins, peut-être, que les questions se focalisent autour d'un point de détail et permettent, par conséquent, de réduire et la multiplicité et la subjectivité des réponses. Cette possibilité, dans la continuité de ce premier texte, devra sans doute être exploitée parallèlement à une analyse basée sur des performances filmées - même si rien ne permet d'affirmer que cette méthode, malgré son ancrage empirique, soit nécessairement efficace ${ }^{9}$. Pour l'heure, tentons seulement d'apporter quelques éléments de réponses aux questions susmentionnées.

Afin de comprendre et de décoder ce type de gestes, chercheurs, musicologues, ethnomusicologues et psychologues de la musique, ont souvent tenté d'appliquer au domaine musical les catégories de la kinésique - des gesture studies ${ }^{10}$. On pense notamment à Jane Davidson ${ }^{11}$ qui, dans ses travaux sur le chant, utilise volontiers la typologie - désormais classique - de Paul Ekman et Wallace V. Friesen ${ }^{12}$; on pense aussi, pour le domaine indien, aux analyses de Martin Clayton $^{13}$ qui s'appuient plus particulièrement sur la typologie de Bernard Rimé et Loris Schiaratura ${ }^{14}$. Vis-à-vis de ces approches, il convient de remarquer trois choses : primo, les typologies sont nombreuses et ne cessent d'être affinées avec l'avancée des recherches et l'essor des gesture studies ${ }^{15}$; secundo, les typologies choisies par Davidson et Clayton le sont essentiellement au regard de leur compatibilité avec la musique étudiée : chant jazz et pop pour la première, chant hindoustani pour le second ; tertio, la difficulté d'adapter ces typologies à la musique va croissant dès lors que l'on s'éloigne du langage parlé : du chant avec paroles, au chant sans paroles, puis à la musique instrumentale. Outre ces tiraillements, liés à la multiplicité des modèles et aux décalages de nature, s'ajoute le problème suivant: tout instrument implique un jeu, une gestuelle et des facultés motrices spécifiques. On remarquera dans le cas des hautbois, et plus généralement des aérophones, l'implication des bras, des mains et des doigts non seulement dans la production du son, mais dans les 
mouvements et les changements de trajectoire de l'instrument. On est loin, par conséquent, des configurations inhérentes au chant où les membres supérieurs sont libres et donc indépendants de la source sonore - de la voix. Aussi me paraitt-il plus judicieux d'élaborer une typologie nouvelle qui, sans ignorer les catégories des gesture studies, évite toute référence directe aux typologies existantes - aucune n'ayant été pensée pour le domaine instrumental. Je propose ainsi de distinguer les mouvements (auxiliaires) pratiques des mouvements (auxiliaires) expressifs - soit deux grandes catégories :

Les mouvements (auxiliaires) pratiques permettent au premier hautboïste de donner aux autres musiciens de la troupe - second hautboïste et/ou percussionnistes - une indication concrète concernant le jeu et son déroulement. Ils renvoient à deux types de mouvements :

Le mouvement qui indique ou annonce un point de départ, une transition, un changement. Il est exécuté par le premier hautboïste et s'adresse au second. Il est plutôt effectué en position 2, côté gauche (côté du partenaire), et peut prendre la forme d'une brève oscillation, tel un « $\mathrm{U}$ » renversé. Ce mouvement est notamment employé dans le kuraippu, partie finale de la forme improvisée et mesurée kalpana-svara ${ }^{16}$, où le premier et le second hautboïstes jouent à tour de rôle en suivant une progression temporelle décroissante au sein de laquelle le nombre de cycles rythmiques (tāla) des séquences qui composent une phase donnée est peu à peu réduit - par exemple: quatre phases respectivement constituées de séquences de $3,2,1$ puis $1 / 2$ cycles de sept temps ${ }^{17}$. Le premier hautboïste, qui a la main, utilise ici ce mouvement en sus des indications sonores (phrasé, parcours mélodico-rythmique, frappe des cymbales, etc.) afin que son partenaire ne manque pas son entrée à la fin de la première séquence de chaque nouvelle phase.

20 Le mouvement correcteur. Il est plutôt exécuté par le premier hautboïste et peut s'adresser à n'importe quel membre de la troupe. Il est effectué en position 2 et s'apparente à une impulsion, souvent brève, vive et répétée, dirigée en direction du musicien concerné. Il est par exemple utilisé pour aider un joueur de cymbales qui aurait perdu la pulsation initiale et/ou le cours du cycle rythmique.

21 Les mouvements (auxiliaires) expressifs s'adressent à l'ensemble des participants : musiciens et auditeurs/spectateurs. Ils suivent le flux mélodico-rythmique et s'adaptent ou non, selon les intuitions, aux émotions que ce flux génère. En ce sens, ils permettent aux joueurs de hautbois de communiquer aux autres musiciens et aux auditeurs ce qu'euxmêmes ressentent lorsqu'ils jouent. On rejoint là, de fait, les suggestions de Vaidyanathan et Ganeshan : ces mouvements, particulièrement en position haute (3), suggèrent non seulement l'empathie, le partage, l'exaltation, mais dévoilent cette volonté toute musicienne, spécialement prégnante dans ce contexte indien, de capter l'attention pour mieux briller. Leur efficacité, pour autant, demeure mystérieuse : elle ne semble résulter ni totalement de leur synchronisation avec la musique, ni totalement de leur caractère individuel - singulier.

On le voit : la gestuelle des joueurs de nägasvaram n'a pas pour seule fonction de captiver ou séduire un auditoire. Elle permet - certes modestement - la coordination des musiciens. Elle permet aussi, plus simplement, de reconnaitre un musicien, de le distinguer des autres à travers cette manière particulière qu'il a de mouvoir, bouger, manipuler, son hautbois - manière qui souligne l'idiosyncrasie de sa gestuelle et au-delà, sans doute, l'unicité du moment. 
23 Il semble pourtant que ce soit là, dans ce dédale infini de figures, que se nichent ces mouvements intentionnels - et expressifs - dont la fonction est effectivement de séduire l'auditoire : cette lente montée que Ganeshan assimile au cobra qui se dresse ; cet au-delà discret de la position haute ( $3 b)$ pratiquée par Subramaniam; ces amples mouvements latéraux préconisés par Vaidyanathan. Règles tacites versus libertés individuelles, intentionnalité versus spontanéité... nous voici donc au cœur d'un vaste réseau dont certaines fonctionnalités paraissent échapper à toute forme de rationalisation. Il n'en reste pas moins qu'un travail passionnant, sur la base des expérimentations et des analyses initiées par Marcelo M. Wanderley (et al.) d'un côtée ${ }^{18}$, et Martin Clayton de l'autre ${ }^{19}$, mérite sans doute d'être développé - au moins pour préciser les rapports que ces mouvements entretiennent avec la musique : hauteurs, mélodies, rythmes, structures, etc.

\section{Épilogue : quand les micros s'en mêlent}

Le son du nāgasvaram rayonne de manière directive, en ligne droite et dans la continuité du corps (long et conique) et du pavillon ${ }^{20}$. Tout changement de trajectoire - et tout mouvement - implique par conséquent une modification du son. Imaginons par exemple un musicien (M) et un auditeur (A), immobile et lui faisant face : si M pointe le pavillon de son instrument en direction de A - a priori en position haute (3) - A perçoit alors le son au maximum de son intensité; si $\mathrm{M}$, en revanche, vise le sol ou les côtés particulièrement en position basse $(1$, surtout a) - A perçoit un son d'intensité plus faible. De la même manière, tout changement de trajectoire engendre une modification du timbre; à cette différence près que la modification est ici essentiellement liée à la trajectoire - la distance n'intervenant pas ou peu dans ce cas. L'effet des gestes auxiliaires n'est donc pas seulement visuel : il est aussi sonore.

On peut dès lors se demander si ces gestes servent effectivement à manipuler le son et si cette manipulation - le cas échéant - vient seconder, doubler ou compléter les techniques habituelles de souffle et de pince. Je ne saurais répondre catégoriquement à cette question dans l'état de mes recherches sinon en soulignant que lorsqu'un joueur de nāgasvaram se dirige peu à peu vers une position haute (3) - donc a priori vers un son soutenu et perçant - l'émotion devient généralement palpable. Ce que semblent confirmer (au plan local) les paroles de Ganeshan et ce que semblent valider (à l'échelle du sous-continent) les observations d'Edward O. Henry sur les liens entre climax émotionnels et intensité/volume sonore dans les traditions musicales indiennes ${ }^{21}$. Quoi qu'il en soit, et quelles que soient les possibilités sonores exploitées par les uns ou les autres, il paraît évident que le contexte au sein duquel les musiciens se produisent est ici un élément déterminant puisqu'il modifie nécessairement, en fonction des lieux et des configurations acoustiques, le rapport des hautboïstes au son, à l'espace et, de fait, à leur propre gestuelle. À cet égard, l'utilisation aujourd'hui systématique des moyens électroacoustiques d'amplification dans les salles de concerts et de mariages est significative ${ }^{22}$. Elle bouleverse en effet le rapport des gestes au son, et vice versa: notre musicien (M) étant dans ce cas tributaire de l'emplacement du micro et notre auditeur (A) de l'orientation des baffles.

Nier cette réalité reviendrait à considérer que les gestes auxiliaires des hautboïstes relèvent uniquement du champ visuel - ce qui est partiellement faux. Mais puisqu'il est question de sens, osons une dernière question, en pensant d'une part à Ganeshan et 
Vaidyanathan, pour qui cette gestuelle est avant tout une affaire de séduction, d'autre part à Alfred Gell, pour qui la musique, comme d'autres formes d'expression, est d'abord une "technologie d'enchantement ${ }^{23}$ »: ces gestes, et les mouvements qu'ils impliquent, ne participeraient-ils pas, en s'adaptant finement aux configurations mélodicorythmiques, aux lieux et aux situations, à ce ravissement soudain qui, l'espace d'un instant, comme par surprise, vient inonder de plaisir les auditeurs et - pour le coup - les musiciens?

\section{BIBLIOGRAPHIE}

CLAYTON, Martin, « Communication in Indian Raga Performance », in MIELL, Dorothy, MACDONALD, Raymond, HARGREAVES, David (ed.), Musical Communication, Oxford, Oxford University Press, 2005, p. 361-381.

CLAYTON, Martin, " Time, Gesture and Attention in a Khyāl Performance », in Asian Music, $\mathrm{N}^{\circ} 38$ (2), 2007, p. 71-96.

DAVIDSON, Jane W., « Bodily Communication in Musical Performance », in MIELL, Dorothy, MACDONALD, Raymond, HARGREAVES, David (ed.), Musical Communication, Oxford, Oxford University Press, 2005, p. 215-237.

DURING, Jean, « Hand made. Pour une anthropologie du geste musical », in Cahiers de musiques traditionnelles, $\mathrm{N}^{\circ} 14,2001$, p. 39-68.

EKMAN, Paul, FRIESEN, Wallace V., " The Repertoire of Nonverbal Behavior : Categories, Origins, Usage, and Coding ", in Semiotica, $\mathrm{N}^{\circ} 1$ (1), 1969, p. 49-98.

FATONE, Gina A., CLAYTON, Martin, LEANTE, Laura, RAHAIM, Matt, « Imagery, Melody and Gesture in Cross-cultural Perspective », in GRITTEN, Anthony, KING, Elaine (ed.),New Perspectives on Music and Gesture, Farnham, Ashgate, 2011, p. 203-220.

GELL, Alfred, « The Technology of Enchantment and the Enchantment of Technology », in COOTE, Jeremy, SHELTON, Anthony (ed.), Anthropology, Art and Aesthetics, Oxford, Clarendon Press, 1992, p. 40-63.

HENRY, Edward O., « The Rationalization of Intensity in Indian Music », inEthnomusicology, $\mathrm{N}^{\circ} 46$ (1), 2002, p. 33-55.

KENDON, Adam, Gesture : Visible Action as Utterance, Cambridge, Cambridge University Press, 2004.

LEANTE, Laura, « The Lotus and the King : Imagery, Gesture and Meaning in a Hindustani Rāg », in Ethnomusicology Forum, № 18 (2), 2009, p. 185-206.

RAHAIM, Matt, « Gesture and Melody in Indian Vocal Music », in Gesture, Nº 8 (3), 2008, p. 325-347.

RIMÉ, Bernard, SCHIARATURA, Loris, « Gesture an Speech », in FELDMAN, Robert S., RIMÉ, Bernard (ed.), Fundamentals of Nonverbal Behavior, Cambridge, Cambridge University Press, 1991, p. 239-281. 
TALLOTTE, William, La voix du serpent. Les sonneurs-batteurs du periya mēlam et le culte āgamique de Śiva : ethnomusicologie d'une pratique musicale au delta de la Kaveri (Tamil Nadu, Inde du Sud), Thèse de doctorat, Université Paris 4-Sorbonne, 2007.

TALLOTTE, William, «L'improvisation comme pratique sociale. L'exemple desnâgasvarakkârar, hautboïstes sud-indiens ", in Tracés, $\mathrm{N}^{\circ} 18,2010$, p. 105-120.

TALLOTTE, William, « L'œil du hautbois. Son, espace et images divines dans le culte śivaïte, Inde du Sud », in Gradhiva, No 15, 2012, p. 202-223.

WANDERLEY, Marcelo M., VINES, Bradley W., « Origins and Functions of Clarinettists' Ancillary Gestures », in GRITTEN, Anthony, KING, Elaine (ed.), Music and Gesture, Aldershot, Ashgate, 2006, p. $165-191$.

WANDERLEY, Marcelo M., VINES, Bradley W., MIDDLETON, Neil, MCKAY, Cory, HATCH, Wesley, «The Musical Significance of Clarinetists' Ancillary Gestures : An Exploration of the Field », in Journal of New Music Research, № 34 (1), 2005, p. 97-113

\section{Discographie}

TALLOTTE, William, Inde du Sud. Periya mēlam. Temple de Chidambaram, 1 CD Ocora/Radio-France C 560178, 2003.

TALLOTTE, William, Inde du Sud. Naiyândi mêlam. Musique des cultes de possession, 1 CD Ocora/RadioFrance C 560245, à paraître.

\section{NOTES}

1. Le nāgasvaram est un grand hautbois sud-indien à perce conique. Joué seul ou en paire, accompagné d'un ou plusieurs tambours tavil, des cymbales tālam et d'un bourdon, il forme un ensemble de sonneurs-batteurs professionnel spécialisé dans le domaine musical savant (karnatique) et connu en pays tamoul sous le nom de periya mèlam ("grand orchestre»). Il accompagne les rituels quotidiens et les fêtes des temples hindous de hautes castes ainsi que les évènements fastes du calendrier: mariages, festivals, meeting politiques, etc. Il est également présent au concert.

2. Terminologie empruntée à Marcelo M. Wanderley et al. : WANDERLEY, Marcelo M., VINES, Bradley W., MIDDLETON, Neil, MCKAY, Cory, HATCH, Wesley, " The Musical Significance of Clarinetists' Ancillary Gestures : An Exploration of the Field », in Journal of New Music Research, № 34 (1), 2005, p. 97-113 ; WANDERLEY, Marcelo M., VINES, Bradley W., «Origins and Functions of Clarinettists' Ancillary Gestures », in GRITTEN, Anthony, KING, Elaine (ed.), Music and Gesture, Aldershot, Ashgate, 2006, p. 165-191.

3. Je pense notamment aux travaux de Martin Clayton, Laura Leante et Matt Rahaim sur la gestuelle des chanteurs hindoustanis, ainsi qu'à ceux de Gina A. Fatone sur les supports gestuels utilisés dans l'apprentissage de la cornemuse écossaise; voir par exemple: CLAYTON, Martin, " Time, Gesture and Attention in a Khyāl Performance », in Asian Music, $\mathrm{N}^{\circ} 38$ (2), 2007, p. 71-96 ; LEANTE, Laura, « The Lotus and the King : Imagery, Gesture and Meaning in a Hindustani Rāg », inEthnomusicology Forum, № 18 (2), 2009, p. 185-206; RAHAIM, Matt, "Gesture and Melody in Indian Vocal Music », in Gesture, No 8 (3), 2008, p. 325-347 ; FATONE, Gina A., CLAYTON, Martin, LEANTE, Laura, RAHAIM, Matt, 
"Imagery, Melody and Gesture in Cross-cultural Perspective ", in GRITTEN, Anthony, KING, Elaine (ed.), New Perspectives on Music and Gesture, Farnham, Ashgate, 2011, p. 203-220.

4. Tableau repris de: TALLOTTE, William, «L'improvisation comme pratique sociale. L'exemple desnâgasvarakkârar, hautboïstes sud-indiens ", in Tracés, $\mathrm{N}^{\circ}$ 18, 2010, p. 117.

5. Sur cette tradition, voir : TALLOTTE, William, Inde du Sud. Naiyândi mêlam. Musique des cultes de possession, 1 CD Ocora/Radio-France C 560245, à paraitre.

6. Cette opposition, entre d'un côté tradition "classique ", temple de hautes castes et gestuelle contenue, et de l'autre tradition "populaire», temple de basses castes et gestuelle ostentatoire, n'est pas sans rappeler - bien que de nature différente - celle évoquée par Jean During entre les gestes des musiciens sédentaires et ceux des musiciens nomades en Asie centrale : DURING, Jean, « Hand made. Pour une anthropologie du geste musical », in Cahiers de musiques traditionnelles, $\mathrm{N}^{\circ}$ 14, 2001, p. 57 et suivantes.

7. S.R.D. Vaidyanathan est aujourd'hui âgé de 76 ans et vit à Chennai (Madras). S'il ne joue plus et n'enseigne plus, il n'en reste pas moins une figure importante du hautbois nāgasvaram et un spécialiste reconnu - et toujours sollicité - du répertoire et de la rythmique karnatiques.

8. Extrait d'entretien repris de: TALLOTTE, William, «L'improvisation comme pratique sociale. L'exemple des nâgasvarakkârar, hautboïstes sud-indiens ", art. cit., p. 117.

9. L'article de Laura Leante, « The Lotus and the King : Imagery, Gesture and Meaning in a Hindustani Rāg ", art. cit., est à cet égard significatif. L'auteur montre en effet, à travers l'analyse d'un geste particulier du bras des chanteurs hindoustanis et le lien de ce geste à un intervalle (re-pa) dans le rāga śrī, que les interprétations et les réalisations des uns et des autres ne se superposent pas toujours. Resserrer le champ des entretiens et de l'analyse ne permet donc pas forcément de résoudre tel ou tel problème.

10. Champ interdisciplinaire de recherche issu de la linguistique et de la psychologie qui s'intéresse aux relations entre parole et gestuelle dans la conversation.

11. Voir par exemple: DAVIDSON, Jane W., «Bodily Communication in Musical Performance ", in MIELL, Dorothy, MACDONALD, Raymond, HARGREAVES, David (ed.), Musical Communication, Oxford, Oxford University Press, 2005, p. 228-231.

12. EKMAN, Paul, FRIESEN, Wallace V., "The Repertoire of Nonverbal Behavior: Categories, Origins, Usage, and Coding ", in Semiotica, $\mathrm{N}^{\circ} 1$ (1), 1969, p. 49-98. Les auteurs proposent dans ce texte une typologie qui distingue: $1 /$ Emblems : gestes ayant un équivalent verbal 2/ Illustrators : gestes illustrant la parole - son sens 3/ Affect displays : gestes révélant les émotions 4/Regulators : gestes indiquant que l'on écoute, comprend, approuve, etc. son interlocuteur 5/Adapters : gestes relevant d'un ajustement corporel.

13. CLAYTON, Martin, "Communication in Indian Raga Performance", in MIELL, Dorothy, MACDONALD, Raymond, HARGREAVES, David (ed.), Musical Communication, Oxford, Oxford University Press, 2005, p. 375 ; CLAYTON, Martin, «Time, Gesture and Attention in a KhyālPerformance », art. cit., p. 74-75 ; FATONE, Gina A., CLAYTON, Martin, LEANTE, Laura, RAHAIM, Matt, "Imagery, Melody and Gesture in Cross-cultural Perspective », art. cit., p. 205.

14. RIMÉ, Bernard, SCHIARATURA, Loris, « Gesture and Speech », in FELDMAN, Robert S., RIMÉ, Bernard (ed.), Fundamentals of Nonverbal Behavior, Cambridge, Cambridge University Press, 1991, p. 239-281. 
15. Pour une histoire des catégories kinésiques au $X X^{e}$ siècle et une approche critique des principales typologies, voir: KENDON, Adam, Gesture: Visible Action as Utterance, Cambridge, Cambridge University Press, 2004, p. 84-107

16. Sur cette forme dans la tradition du periya mèlam, voir : TALLOTTE, William, La voix du serpent. Les sonneurs-batteurs du periya mēlam et le culte āgamique de Śiva : ethnomusicologie d'une pratique musicale au delta de la Kaveri (Tamil Nadu, Inde du Sud), Thèse de doctorat, Université Paris 4-Sorbonne, 2007, p. 243-247.

17. Voir: TALLOTTE, William, Inde du Sud. Periya mēlam. Temple de Chidambaram, $1 \mathrm{CD}$ Ocora/Radio-France C 560178, 2003, plage 2, de 16'51 (ou 17’09) à 18'25. Le départ du kuraippuest ici sujet à discussion : débute-t-il en effet à 16'51, départ initialement prévu par le premier hautboïste mais manqué par le second, qui joue alors sur 4 cycles au lieu de 3 , ou à 17'09, lorsque le duo est parfaitement coordonné ? Pour une transcription de ce passage, voir notre thèse, p.328-329; le minutage renvoie alors au CD d'accompagnement : CD1, plage 3, de 16'52 (ou 17'10) à 18'26.

18. Voir note 2.

19. Voir notes 3 et 13.

20. Sur la directivité du hautbois nāgasvaram et l'utilisation de cette caractéristique en contexte rituel, voir: TALLOTTE, William, «L'œil du hautbois. Son, espace et images divines dans le culte śivaïte, Inde du Sud », in Gradhiva, n 15, 2012, p. 214-219.

21. HENRY, Edward O., "The Rationalization of Intensity in Indian Music», in Ethnomusicology, $\mathrm{N}^{\circ} 46$ (1), 2002, p. 48 et précédentes.

22. Le temple n'est pas toujours épargné par ces dispositifs. Il arrive notamment, lors des plus grandes fêtes, que le départ des grandes processions soit sonorisé.

23. Voir GELL, Alfred, "The Technology of Enchantment and the Enchantment of Technology ", in COOTE, Jeremy, SHELTON, Anthony (ed.), Anthropology, Art and Aesthetics, Oxford, Clarendon Press, p. 40-63.

\section{RÉSUMÉS}

Le jeu du hautbois nāgasvaram fait appel à des gestes qui n'interviennent pas directement dans la production du son : l'instrument, en effet, est régulièrement mis en mouvement, verticalement, horizontalement, ou en combinant ces deux plans. Si les musiciens ne parlent généralement pas de ces gestes et des mouvements qu'ils impliquent, ils laissent parfois entendre que leur fonction est de capter l'attention des auditeurs/spectateurs, voire de les séduire. Cet article, à partir d'enquêtes de terrain menées au Tamil Nadu, se penche sur les codes qui sous-tendent ces gestes et le rôle, voire la signification, que l'on peut raisonnablement leur accorder en différentes situations de performance.

Playing the nāgasvaram shawm involves gestures that are not directly involved in sound production: the instrument is indeed regularly set in motion, vertically, horizontally, or by combining these two planes. Musicians do not generally speak of these gestures and the movements they imply, but they suggest, sometimes, that their function is to gain the attention 
of the auditors/spectators, and even to seduce them. This article, from fieldwork carried out in Tamil Nadu, looks into the codes that underlie these gestures and the role, even the meaning, which we can reasonably grant them in various performance situations.

INDEX

Mots-clés : attention, ethnomusicologie, geste instrumental, hautbois nāgasvaram, Inde du Sud, kinésique

Keywords : attention, ethnomusicology, instrumental gesture, nāgasvaram shawm, South India, gesture studies

\section{AUTEUR}

\section{WILLIAM TALLOTTE}

William Tallotte est ethnomusicologue et travaille depuis une quinzaine d'années sur les pratiques musicales du pays tamoul (Inde du Sud). Il a soutenu une thèse de doctorat en décembre 2007 à l'Université Paris-Sorbonne (Paris 4). Il a enseigné dans plusieurs universités françaises (Bordeaux 3, Strasbourg 2 et Rennes 2) et a été post-doctorant au musée du quai Branly (2009-2010). Il est actuellement chercheur associé au Centre de Recherche Patrimoines et Langages Musicaux (PLM, EA 4087). 